\title{
Intuitionistic Trapezoidal Fuzzy Group Decision-Making Based on Prospect Choquet Integral Operator and Grey Projection Pursuit Dynamic Cluster
}

\author{
Jiahang Yuan and Cunbin Li \\ School of Economics and Management, North China Electric Power University, Beijing 102206, China \\ Correspondence should be addressed to Jiahang Yuan; yuanjiahang@126.com
}

Received 7 November 2016; Accepted 18 December 2016; Published 20 February 2017

Academic Editor: Kishin Sadarangani

Copyright (C) 2017 Jiahang Yuan and Cunbin Li. This is an open access article distributed under the Creative Commons Attribution License, which permits unrestricted use, distribution, and reproduction in any medium, provided the original work is properly cited.

\begin{abstract}
In consideration of the interaction among attributes and the influence of decision makers' risk attitude, this paper proposes an intuitionistic trapezoidal fuzzy aggregation operator based on Choquet integral and prospect theory. With respect to a multiattribute group decision-making problem, the prospect value functions of intuitionistic trapezoidal fuzzy numbers are aggregated by the proposed operator; then a grey relation-projection pursuit dynamic cluster method is developed to obtain the ranking of alternatives; the firefly algorithm is used to optimize the objective function of projection for obtaining the best projection direction of grey correlation projection values, and the grey correlation projection values are evaluated, which are applied to classify, rank, and prefer the alternatives. Finally, an illustrative example is taken in the present study to make the proposed method comprehensible.
\end{abstract}

\section{Introduction}

Multiattribute group decision-making (MAGDM) provides a systematic quantitative approach for decision-making problems involving multiple attributes and actions and can assist decision makers (DMs) in rationally considering all the important objective and subjective attributes of a problem [1, $2]$. With the complexity of social environment and boundedness of human cognition, it is difficult to use crisp values to characterize the evaluation information. Fuzzy sets (FS) are generalized to model the vagueness in decision evaluation. But, a fuzzy set has limitation on presenting a broad description of all information related to the specific decision problem under an uncertain environment [3]. In 1965, Atanassov put forward the idea of intuitionistic fuzzy sets (IFS) which contained membership function and nonmembership function that represented the decision maker's supporting degree and opposition degree. Owed to the capacity of expressing the fuzziness and hesitancy originating from imprecise knowledge or information, scholars have made many achievements in MAGDM problems with IFS, such as traditional decision-making methods with new definitions, new hybrid decision-making methods, extension of distance measure, and aggregation operators. As the combination of traditional fuzzy numbers and IFS, the intuitionistic fuzzy number (IFN) based on real numbers is a good match for describing an ill-known quantity. In recent years, the studies focused on three kinds of IFNs: interval-valued intuitionistic fuzzy number, intuitionistic triangular fuzzy number, and intuitionistic trapezoidal fuzzy number (ITFN).

Chen et al. (2016) proposed four aggregation operators based on interval-valued intuitionistic fuzzy numbers and made the experimental results to show the drawbacks of existing methods [4]. As the extension of triangular intuitionistic fuzzy numbers, ITFNs have the better capability to contain rich fuzzy information. Yuan and $\mathrm{Li}$ (2016) defined intuitionistic trapezoidal fuzzy random variable based on ITFNs and proposed decision-making method based on MahalanobisTaguchi Gram-Schmidt and evidence theory [5]. Lakshmana et al. (2016) proposed a linear ordering class method to rank the alternatives in MADM with ITFNs [6]. Kumar et al. (2015) utilized the shortest path as a classical network optimization method to classify alternatives by finding the shortest path and shortest distance [7]. Wu and Cao (2013) 
presented some cases of aggregation operators with intuitionistic trapezoidal fuzzy numbers and then applied the ITFWG and the ITFHG operators to MADM problem [8]. Based on Wu and Cao's research, Wan and Dong (2015) investigated four kinds of power geometric operators of ITFNs and developed four MADM methods for different cases [9]. Motivated by the aggregation ability of operators, Yuan et al. (2016) proposed a new aggregation method for interval-valued intuitionistic hesitant fuzzy information based on Choquet integral [10]. Chen and Chang (2016) established transformation techniques between intuitionistic fuzzy values and right-angled triangular fuzzy numbers and proposed new geometric averaging operators to deal with ITFNs [11].

To sum up, aggregation operators are effective tools to aggregate ITFNs, but the existing research has some drawbacks.

(1) Some decision methods use subjective weight determination methods. This approach would add the fuzziness and incongruence in decision information.

(2) Most aggregation operators do not consider the interdependency characteristics of attributes, but the assumption of independency of attribute is too strong to be satisfied in many MADM problems.

(3) The researches premises are formed commonly based on the condition that the decision makers are absolutely reasonable, whereas actually the subjective psychological factors and attitude towards risk in decision leaders directly influence the risk evaluation results.

(4) In group decision-making, the construction of decision maker evaluation system often needs to meet the requirement of completeness, representativeness, and independence. However, it is slightly harsh to meet the requirement of independence in the actual decision-making problems. Preferences of decision makers are often affected by status, prestige, knowledge, and other factors.

In view of studying the existing literatures, this paper proposes an intuitionistic trapezoidal fuzzy aggregation operator based on Choquet integral in consideration of the validity of fuzzy measure. Combined with prospect theory, the ITFN prospect value function and the intuitionistic trapezoidal fuzzy Choquet integral prospect operator are proposed. Aiming at the MAGDM problem with ITFNs, this paper presents grey relation-projection pursuit dynamic cluster method based on intuitionistic trapezoidal fuzzy Choquet integral prospect operator. Through history information, the fuzzy measures of decision makers are obtained. The intuitionistic trapezoidal fuzzy numbers are aggregated by the proposed operator, and then the alternatives ranking is obtained by grey relation with projection pursuit dynamic cluster method. The remainder of this paper unfolds as follows: Section 2 introduces some basic definitions of intuitionistic trapezoidal fuzzy number. The aggregation operator based on Choquet integral and prospect theory is developed in Section 3. Section 4 shows the decision-making method. In Section 5, an example and its comparison analysis are given. Section 6 ends the paper with some concluding remarks.

The main contributions of this paper are as follows.

(1) An intuitionistic trapezoidal fuzzy aggregation Choquet integral operator is proposed to eliminate the interdependence between attributes.

(2) The prospect value function for ITFNs is defined in consideration of different risk attitude.

(3) In order to avoid subjective weights and information loss, a grey relation-projection pursuit dynamic cluster method based on firefly algorithm is developed to rank alternatives.

\section{Intuitionistic Trapezoidal Fuzzy Number}

Definition 1 (see [5]). Let $\widetilde{A}=\left\langle\left([a, b, c, d] ; \mu_{A}\right),\left(\left[a^{\prime}, b, c, d^{\prime}\right]\right.\right.$; $\left.\left.v_{\widetilde{A}}\right)\right\rangle$ be an intuitionistic trapezoidal fuzzy number in the universe of discourse $X$, whose membership function and nonmembership function are defined as follows:

$$
\begin{aligned}
& \mu_{\widetilde{A}}(x)= \begin{cases}\frac{x-a}{b-a} \mu_{\widetilde{A^{\prime}}}, & \text { if } a \leq x<b, \\
\mu_{\widetilde{A}}, & \text { if } b \leq x \leq c, \\
\frac{d-x}{d-c} \mu_{\widetilde{A^{\prime}}}, & \text { if } c<x \leq d, \\
0, & \text { if } x<a \text { or } x>d,\end{cases} \\
& v_{\widetilde{A}}(x)= \begin{cases}\frac{b-x+\left(x-a^{\prime}\right) v_{\widetilde{A}},}{b-a^{\prime}} & \text { if } a^{\prime} \leq x<b, \\
v_{\widetilde{A}}, & \text { if } b \leq x \leq c, \\
\frac{x-c+\left(d^{\prime}-x\right) v_{\widetilde{A}},}{d^{\prime}-c} & \text { if } c<x \leq d^{\prime}, \\
1, & \text { if } x<a^{\prime} \text { or } x>d^{\prime},\end{cases}
\end{aligned}
$$

respectively, where $a, b, c, d, a^{\prime}, d^{\prime}$ are real numbers and $0 \leq$ $\mu_{\widetilde{A}} \leq 1,0 \leq v_{\widetilde{A}} \leq 1,0 \leq \mu_{\widetilde{A}}+v_{\widetilde{A}} \leq 1$. If $a=a^{\prime}, d=d^{\prime}$, the intuitionistic trapezoidal fuzzy number is simplified to $\widetilde{A}=$ $\left([a, b, c, d] ; \mu_{\widetilde{A}}, v_{\widetilde{A}}\right)$ which is the study content of this paper.

Definition 2. Let $\widetilde{A}_{1}=\left(\left[a_{1}, b_{1}, c_{1}, d_{1}\right] ; \mu_{A_{1}}, v_{\widetilde{A}_{1}}\right)$ and $\widetilde{A}_{2}=\left(\left[a_{2}\right.\right.$, $\left.\left.b_{2}, c_{2}, d_{2}\right] ; \mu_{A_{2}}, v_{A_{2}}\right)$ be two intuitionistic trapezoidal fuzzy numbers in the universe of discourse $X$. The Hamming distance between $\widetilde{A}_{1}$ and $\widetilde{A}_{2}$ is

$$
\begin{aligned}
d & \left(\widetilde{A}_{1}, \widetilde{A}_{2}\right) \\
& =\frac{1}{8}\left(\left|\left(1+\mu_{\widetilde{A_{1}}}-v_{\widetilde{A_{1}}}\right) a_{1}-\left(1+\mu_{\widetilde{A_{2}}}-v_{\widetilde{A_{2}}}\right) a_{2}\right|\right. \\
& +\left|\left(1+\mu_{\widetilde{A_{1}}}-v_{\widetilde{A_{1}}}\right) b_{1}-\left(1+\mu_{\widetilde{A_{2}}}-v_{\widetilde{A_{2}}}\right) b_{2}\right| \\
& +\left|\left(1+\mu_{\widetilde{A_{1}}}-v_{\widetilde{A_{1}}}\right) c_{1}-\left(1+\mu_{\widetilde{A_{2}}}-v_{\widetilde{A_{2}}}\right) c_{2}\right| \\
& \left.+\left|\left(1+\mu_{\widetilde{A_{1}}}-v_{\widetilde{A_{1}}}\right) d_{1}-\left(1+\mu_{\widetilde{A_{2}}}-v_{\widetilde{A_{2}}}\right) d_{2}\right|\right) .
\end{aligned}
$$


Definition 3. Let $\widetilde{A}_{1}=\left(\left[a_{1}, b_{1}, c_{1}, d_{1}\right] ; \mu_{A_{1}}, v_{A_{1}}\right)$ and $\widetilde{A}_{2}=$ $\left(\left[a_{2}, b_{2}, c_{2}, d_{2}\right] ; \mu_{\widetilde{A}_{2}}, v_{A_{2}}\right)$ be two intuitionistic trapezoidal fuzzy numbers in the universe of discourse $X . T_{\widetilde{A}_{1}}=\left[a_{1}, b_{1}\right.$, $\left.c_{1}, d_{1}\right]$ and $T_{\widetilde{A}_{2}}=\left[a_{2}, b_{2}, c_{2}, d_{2}\right]$ are traditional trapezoidal fuzzy numbers and $\left\|T_{A_{1}}^{\lambda}\right\|=\left|\left((1-\lambda)\left(a_{1}+b_{1}\right)+\lambda\left(c_{1}+d_{1}\right)\right) / 4\right|$ and $\left\|T_{A_{2}}^{\lambda}\right\|=\left|\left((1-\lambda)\left(a_{2}+b_{2}\right)+\lambda\left(c_{2}+d_{2}\right)\right) / 4\right|$. The algorithms of intuitionistic trapezoidal fuzzy numbers are as follows.

(1) Addition:

$$
\begin{aligned}
& \widetilde{A}_{1} \oplus \widetilde{A}_{2}=\left(\left[a_{1}+a_{2}, b_{1}+b_{2}, c_{1}+c_{2}, d_{1}+d_{2}\right] ;\right.
\end{aligned}
$$

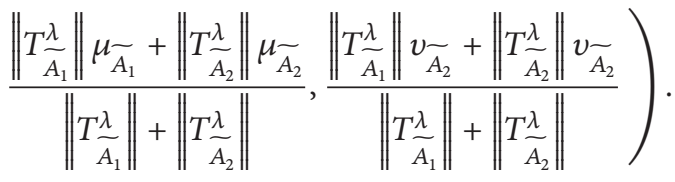

(2) Subtraction:

$$
\begin{aligned}
& \widetilde{A}_{1} \ominus \widetilde{A}_{2}=\left(\left[a_{1}-d_{2}, b_{1}-c_{2}, c_{1}-b_{2}, d_{1}-a_{2}\right] ;\right.
\end{aligned}
$$

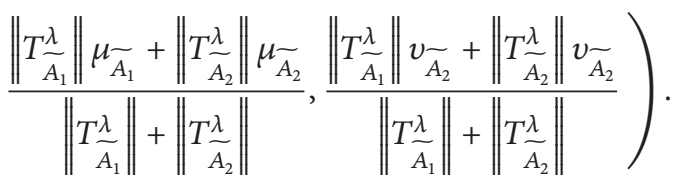

From the algorithms, we find that

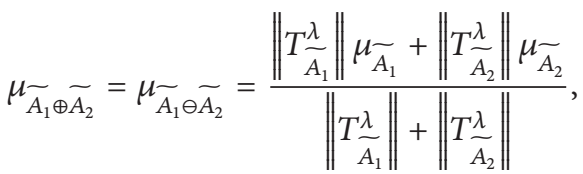

$$
\begin{aligned}
& v_{\widetilde{A_{1}} \widetilde{A}_{2}}=v_{\widetilde{A_{1}} \odot \widetilde{A_{2}}}=\frac{\left\|T_{\widetilde{A_{1}}}^{\lambda}\right\| v_{\widetilde{A_{1}}}+\left\|T \frac{T_{A_{2}}}{\|}\right\| v_{\widetilde{A_{2}}}}{\left\|T_{\widetilde{A_{1}}}^{\lambda}\right\|+\left\|T \frac{\lambda}{\widetilde{A}_{2}}\right\|} .
\end{aligned}
$$

(3) Multiplication:

$$
\begin{aligned}
\widetilde{A}_{1} & \otimes \widetilde{A}_{2}=\left(\left[\min \left(a_{1} a_{2}, a_{1} d_{2}, d_{1} a_{2}, d_{1} d_{2}\right),\right.\right. \\
& \min \left(b_{1} b_{2}, b_{1} c_{2}, c_{1} b_{2}, c_{1} c_{2}\right), \max \left(b_{1} b_{2}, b_{1} c_{2}, c_{1} b_{2}, c_{1} c_{2}\right), \\
& \left.\max \left(a_{1} a_{2}, a_{1} d_{2}, d_{1} a_{2}, d_{1} d_{2}\right)\right] ; \mu_{A_{1}} \mu_{\widetilde{A}_{2}}, v_{\widetilde{A}_{1}}+v_{\widetilde{A}_{2}} \\
& \left.-v_{\widetilde{A}_{1}} v_{\widetilde{A}_{2}}\right) .
\end{aligned}
$$

(4) Scalar-multiplication:

$$
\chi \widetilde{A}_{1}= \begin{cases}\left(\left[\chi a_{1}, \chi b_{1}, \chi c_{1}, \chi d_{1}\right] ; \mu_{\widetilde{A}_{1}}, v_{\widetilde{A_{1}}}\right), & \chi \geq 0, \\ \left(\left[\chi d_{1}, \chi c_{1}, \chi b_{1}, \chi a_{1}\right] ; \mu_{\widetilde{A_{1}}}, v_{\widetilde{A_{1}}}\right), & \chi<0 .\end{cases}
$$

(5) Exponentiation:

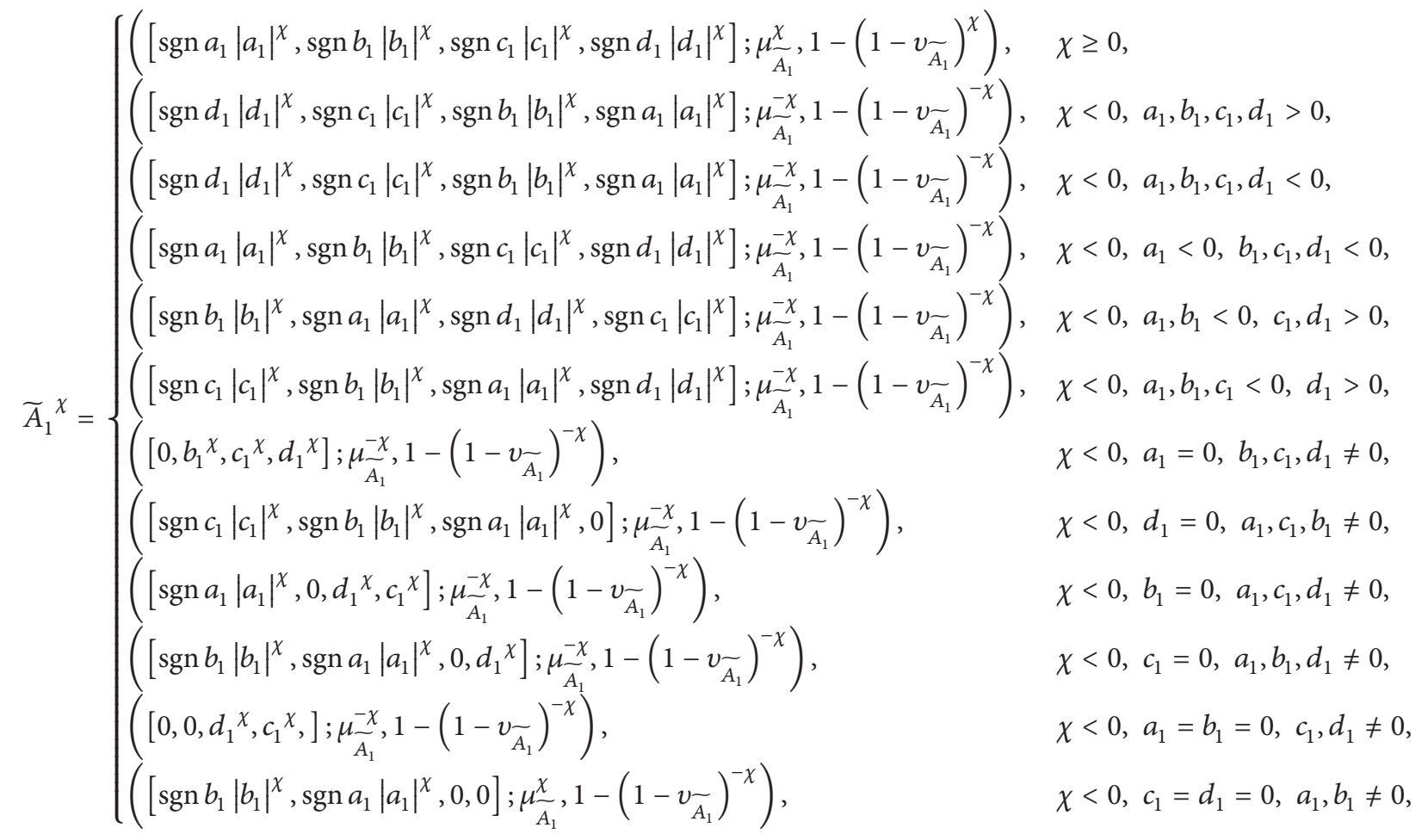


where sgn is a sign function,

$$
\operatorname{sgn} x= \begin{cases}1, & x>0, \\ 0, & x=0, \quad x \in R . \\ -1, & x<0,\end{cases}
$$

(6) Division. If $a_{2}, b_{2}, c_{2}, d_{2} \neq 0$, then

$$
\begin{aligned}
& \frac{\widetilde{A}_{1}}{\widetilde{A}_{2}}=\left(\left[\min \left(\frac{a_{1}}{a_{2}}, \frac{a_{1}}{d_{2}}, \frac{d_{1}}{a_{2}}, \frac{d_{1}}{d_{2}}\right),\right.\right. \\
& \min \left(\frac{b_{1}}{b_{2}}, \frac{b_{1}}{c_{2}}, \frac{c_{1}}{b_{2}}, \frac{c_{1}}{c_{2}}\right), \max \left(\frac{b_{1}}{b_{2}}, \frac{b_{1}}{c_{2}}, \frac{c_{1}}{b_{2}}, \frac{c_{1}}{c_{2}}\right), \\
& \left.\max \left(\frac{a_{1}}{a_{2}}, \frac{a_{1}}{d_{2}}, \frac{d_{1}}{a_{2}}, \frac{d_{1}}{d_{2}}\right)\right] ; \mu \widetilde{A_{1}} \mu_{A_{2}}, v_{\widetilde{A_{1}}}+v_{\widetilde{A_{2}}} \\
& \left.-v_{\widetilde{A_{1}}} v_{\widetilde{A_{2}}}\right) .
\end{aligned}
$$

\section{Aggregation Operators}

\subsection{Intuitionistic Trapezoidal Fuzzy Choquet Integral (ITFCI)}

Definition 4 (see [13]). Suppose that $\mu$ is the fuzzy measure in the universe of discourse $X=\left\{x_{1}, x_{2}, \ldots, x_{n}\right\}$ and $\widetilde{A}_{i}=\left(\left[a_{i}\right.\right.$, $\left.\left.b_{i}, c_{i}, d_{i}\right] ; \mu_{i}, v_{i}\right)(i=1,2, \ldots, n)$ are intuitionistic trapezoidal fuzzy numbers based on $X$. Then the discrete Choquet integral of fuzzy measure $\mu$ is defined as follows:

$$
\begin{aligned}
\int \widetilde{A} d u= & \operatorname{ITFCI}\left(\widetilde{A}_{1}, \widetilde{A}_{2}, \ldots, \widetilde{A}_{n}\right) \\
= & \widetilde{A}_{\tau(1)}\left[\mu\left(B_{\tau(1)}\right)-\mu\left(B_{\tau(2)}\right)\right] \oplus \cdots \\
& \oplus \widetilde{A}_{\tau(n)}\left[\mu\left(B_{\tau(n)}\right)-\mu\left(B_{\tau(n+1)}\right)\right] \\
= & \sum_{i=1}^{n} \widetilde{A}_{\tau(i)}\left[\mu\left(B_{\tau(i)}\right)-\mu\left(B_{\tau(i+1)}\right)\right],
\end{aligned}
$$

where $\left(\widetilde{A}_{\tau(1)}, \widetilde{A}_{\tau(2)}, \ldots, \widetilde{A}_{\tau(n)}\right)$ is the permutation of $\left(\widetilde{A}_{1}, \widetilde{A}_{2}\right.$, $\left.\ldots, \widetilde{A}_{n}\right)$, for any $i, \widetilde{A}_{\tau(i)} \leq \widetilde{A}_{\tau(i+1)} ; B_{\tau(i)}=\left(x_{\tau(i)}, x_{\tau(i+1)}, \ldots\right.$, $\left.x_{\tau(n)}\right), B_{\tau(n+1)}=0$.

Theorem 5. Let $\widetilde{A}_{i}=\left(\left[a_{i}, b_{i}, c_{i}, d_{i}\right] ; \mu_{i}, v_{i}\right)(i=1,2, \ldots, n)$ be a set of intuitionistic trapezoidal fuzzy numbers; $\mu$ is the fuzzy measure in the universe of discourse $X=\left\{x_{1}, x_{2}, \ldots, x_{n}\right\}$; then $\operatorname{ITFCI}\left(\widetilde{A}_{1}, \widetilde{A}_{2}, \ldots, \widetilde{A}_{n}\right)$ is still a set of intuitionistic trapezoidal fuzzy numbers. And the following equation is valid:

$$
\begin{gathered}
\operatorname{ITFCI}\left(\widetilde{A}_{1}, \widetilde{A}_{2}, \ldots, \widetilde{A}_{n}\right) \\
=\left(\left[\sum_{i=1}^{n}\left[\mu\left(B_{\tau(i)}\right)-\mu\left(B_{\tau(i+1)}\right)\right] a_{\tau(i)},\right.\right. \\
\sum_{i=1}^{n}\left[\mu\left(B_{\tau(i)}\right)-\mu\left(B_{\tau(i+1)}\right)\right] b_{\tau(i)},
\end{gathered}
$$

$$
\begin{aligned}
& \sum_{i=1}^{n}\left[\mu\left(B_{\tau(i)}\right)-\mu\left(B_{\tau(i+1)}\right)\right] c_{\tau(i)}, \\
& \left.\sum_{i=1}^{n}\left[\mu\left(B_{\tau(i)}\right)-\mu\left(B_{\tau(i+1)}\right)\right] d_{\tau(i)}\right] ; \frac{\sum_{i=1}^{n}\left\|\widetilde{A}_{\tau(i)}\right\| \mu_{\tau(i)}}{\sum_{i=1}^{n}\left\|\widetilde{A}_{\tau(i)}\right\|}, \\
& \left.\frac{\sum_{i=1}^{n}\left\|\widetilde{A}_{\tau(i)}\right\| \nu_{\tau(i)}}{\sum_{i=1}^{n}\left\|\widetilde{A}_{\tau(i)}\right\|}\right) .
\end{aligned}
$$

The operator occupies some properties, idempotent, monotonic, and limited.

Proposition 6 (idempotency). Let $\widetilde{A}_{i}=\left(\left[a_{i}, b_{i}, c_{i}, d_{i}\right] ; \mu_{i}\right.$, $\left.v_{i}\right)(i=1,2, \ldots, n)$ be a set of intuitionistic trapezoidal fuzzy numbers; $\mu$ is the fuzzy measure in the universe of discourse $X=\left\{x_{1}, x_{2}, \ldots, x_{n}\right\}$. If $\widetilde{A}_{1}=\widetilde{A}_{2}=\cdots \widetilde{A}_{n}=\widetilde{A}=$ $\left([a, b, c, d] ; \mu_{\widetilde{A}}, v_{\widetilde{A}}\right)$; then $\operatorname{ITFCI}\left(\widetilde{A}_{1}, \widetilde{A}_{2}, \ldots, \widetilde{A}_{n}\right)=\widetilde{A}$.

Proposition 7 (monotonicity). Let $\widetilde{A}_{i}=\left(\left[a_{i}, b_{i}, c_{i}, d_{i}\right] ; \mu_{i}\right.$, $\left.v_{i}\right)(i=1,2, \ldots, n)$ and $\widetilde{A}_{i}^{\prime}=\left(\left[a_{i}^{\prime}, b_{i}^{\prime}, c_{i}^{\prime}, d_{i}^{\prime}\right] ; \mu_{i}^{\prime}, v_{i}^{\prime}\right)(i=$ $1,2, \ldots, n)$ be two sets of intuitionistic trapezoidal fuzzy numbers; $\mu$ is the fuzzy measure in the universe of discourse $X=$ $\left\{x_{1}, x_{2}, \ldots, x_{n}\right\}$. If $\left(\widetilde{A}_{\tau(1)}, \widetilde{A}_{\tau(2)}, \ldots, \widetilde{A}_{\tau(n)}\right)$ and $\left(\widetilde{A}_{\tau(1)}^{\prime}, \widetilde{A}_{\tau(2)}^{\prime}\right.$, $\left.\ldots, \widetilde{A}_{\tau(n)}^{\prime}\right)$ are the permutations of $\left(\widetilde{A}_{1}, \widetilde{A}_{2}, \ldots, \widetilde{A}_{n}\right)$ and $\left(\widetilde{A}_{1}^{\prime}\right.$, $\left.\widetilde{A}_{2}^{\prime}, \ldots, \widetilde{A}_{n}^{\prime}\right)$, respectively, then we have the following relationship: $\widetilde{A}_{\tau(i)} \leq \widetilde{A}_{\tau(i+1)}, \widetilde{A}_{\tau(i)}^{\prime} \leq \widetilde{A}_{\tau(i+1)}^{\prime}$. For any $i, \widetilde{A}_{\tau(i)} \geq \widetilde{A}_{\tau(i)}^{\prime}$ corresponds to $a_{\tau(i)} \geq a_{\tau(i)}^{\prime}, b_{\tau(i)} \geq b_{\tau(i)}^{\prime}, c_{\tau(i)} \geq c_{\tau(i)}^{\prime}, d_{\tau(i)} \geq d_{\tau(i)}^{\prime}$, $\mu_{\tau(i)} \geq \mu_{\tau(i)}^{\prime}$, but $v_{\tau(i)} \geq v_{\tau(i)}^{\prime}$. Then $\operatorname{ITFCI}\left(\widetilde{A}_{1}, \widetilde{A}_{2}, \ldots \widetilde{A}_{n}\right) \geq$ $\operatorname{ITFCI}\left(\widetilde{A}_{1}^{\prime}, \widetilde{A}_{2}^{\prime}, \ldots, \widetilde{A}_{n}^{\prime}\right)$

Proposition 8 (boundedness). Let $\widetilde{A}_{i}=\left(\left[a_{i}, b_{i}, c_{i}, d_{i}\right] ; \mu_{i}\right.$, $\left.v_{i}\right)(i=1,2, \ldots, n)$ be a set of intuitionistic trapezoidal fuzzy numbers; $\mu$ is the fuzzy measure in the universe of discourse $X=\left\{x_{1}, x_{2}, \ldots, x_{n}\right\}$. Suppose that $\widetilde{A}^{-}=$ $\left(\left[\min _{i} a_{i}, \min _{i} b_{i}, \min _{i} c_{i}, \min _{i} d_{i}\right] ; \min _{i} \mu_{i}, \min _{i} v_{i}\right)$ and $\widetilde{A}^{+}=$ $\left(\left[\max _{i} a_{i}, \max _{i} b_{i}, \max _{i} c_{i}, \max _{i} d_{i}\right] ; \max _{i} \mu_{i}, \max _{i} v_{i}\right)$; then $\widetilde{A}^{-} \leq$ $\operatorname{ITFCI}\left(\widetilde{A}_{1}, \widetilde{A}_{2}, \ldots, \widetilde{A}_{n}\right) \leq \widetilde{A}^{+}$.

3.2. Intuitionistic Trapezoidal Fuzzy Choquet Integral Prospect Operator. For the problem of subjective risk preference, Kahneman and Tversky proposed prospect theory abstracted from the behavior principle of decision maker in the actual decision-making process in 1979. It explains the phenomenon that expected utility theory cannot be explained, such as Allais Paradox and Ellsberg Paradox. Prospect theory reveals the selection decision model under uncertain conditions, points out the risk attitudes and sensibility when decision makers confronted benefit or cost, and has some characteristics such as diminishing marginal utility, reliance on reference point, and loss aversion. Therefore, the combination of the intuitionistic trapezoidal fuzzy Choquet integral (ITFCI) operator with prospect theory is reasonable and factual in information aggregation. 
Definition 9. Let $\widetilde{A}_{i}=\left(\left[a_{i}, b_{i}, c_{i}, d_{i}\right] ; \mu_{i}, v_{i}\right)(i=1,2, \ldots, n)$ be a set of intuitionistic trapezoidal fuzzy numbers. Let $\widetilde{R}=$ $([a, b, c, d] ; \mu, v)$ be the reference point based on prospect theory, where $\mu$ is the expected membership function and $v$ is the expected nonmembership function. Then the deviation degree between $\widetilde{A}_{i}$ and $\widetilde{R}$ is

$$
\begin{aligned}
\Delta \widetilde{A}_{i} & =\widetilde{A}_{i} \ominus \widetilde{R} \\
& =\left(\left[a_{i}-d, b_{i}-c, c_{i}-b, d_{i}-a\right] ; \mu_{\widetilde{A \ominus \widetilde{R}}}, v_{\widetilde{A} \odot \widetilde{R}}\right),
\end{aligned}
$$

where

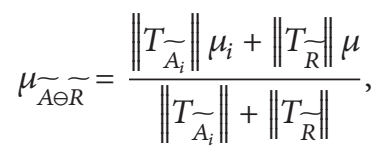

$$
\begin{aligned}
& v_{\widetilde{A} \odot \widetilde{R}}=\frac{\left\|T_{\mathcal{A}_{i}}\right\| v_{i}+\left\|T_{\widetilde{R}}\right\| v}{\left\|T_{\widetilde{A_{i}}}\right\|+\left\|T_{\widetilde{R}}\right\|}, \\
& \left\|T_{\widetilde{A_{i}}}\right\|=\frac{\left(\left|a_{i}\right|+\left|b_{i}\right|+\left|c_{i}\right|+\left|d_{i}\right|\right)}{4}, \\
& \left\|T_{\widetilde{R}}\right\|=\frac{(|a|+|b|+|c|+|d|)}{4} .
\end{aligned}
$$

Definition 10. Let $\Delta \widetilde{A}_{i}=\left(\left[a_{i}-d, b_{i}-c, c_{i}-b, d_{i}-a\right] ; \mu \widetilde{A \ominus \widetilde{R}}\right.$, $\left.v_{\widetilde{A \ominus R}}\right)$ be a set of intuitionistic trapezoidal fuzzy deviation degree; then the intuitionistic trapezoidal fuzzy prospect value function of $\widetilde{A}_{i}$ is

$$
\begin{aligned}
& v\left(\Delta \widetilde{A}_{i}\right)
\end{aligned}
$$

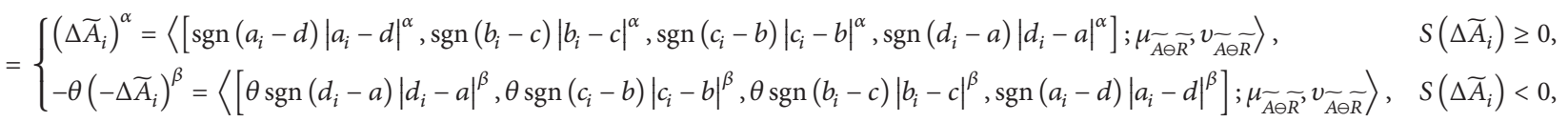

where $\Delta \widetilde{A}_{i}$ is the deviation degree between $\widetilde{A}_{i}$ and $\widetilde{R}, S\left(\Delta \widetilde{A}_{i}\right)$ is the score function of $\Delta \widetilde{A}_{i}$, and $S\left(\Delta \widetilde{A}_{i}\right)=1 / 4 \times\left(a_{i}-d+b_{i}-c+\right.$

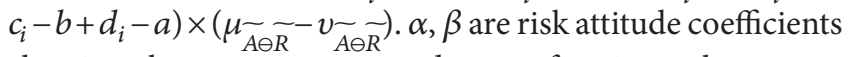
showing the concave-convex degree of region value power function of the gains and the losses and $0 \leq \alpha, \beta \leq 1, \theta$ is loss aversion coefficient which represents that the region value function is steeper for the losses than the gains.

Generally, the prospect value is composed of value function and weights. However, in the process of determining the weights, if the decision makers are required to provide the subjective probability repeatedly, it is easy to distort the result due to excessive subjective judgement. Thus, drawing the experience from [13], this paper calculates the weights based on fuzzy measure and defines the intuitionistic trapezoidal fuzzy Choquet integral prospect (ITFCIP) operator.

Definition 11. Suppose that $\mu$ is the fuzzy measure in the universe of discourse $X=\left\{x_{1}, x_{2}, \ldots, x_{n}\right\}$ and $v\left(\Delta \widetilde{A}_{i}\right)$ is a set of intuitionistic trapezoidal fuzzy prospect value function of $\widetilde{A}_{i}=\left(\left[a_{i}, b_{i}, c_{i}, d_{i}\right] ; \mu_{i}, v_{i}\right)(i=1,2, \ldots, n)$. Then the discrete Choquet integral of $v\left(\Delta \widetilde{A}_{i}\right)$ on fuzzy measure $\mu$ is defined as follows:

$$
\begin{aligned}
& \int v\left(\Delta \widetilde{A}_{i}\right) d u \\
& =\operatorname{ITFCI}\left(v\left(\Delta \widetilde{A}_{1}\right), v\left(\Delta \widetilde{A}_{2}\right), \ldots, v\left(\Delta \widetilde{A}_{n}\right)\right) \\
& =v\left(\Delta \widetilde{A}_{\tau(1)}\right)\left[\mu\left(B_{\tau(1)}\right)-\mu\left(B_{\tau(2)}\right)\right] \oplus \cdots \\
& \quad \oplus v\left(\Delta \widetilde{A}_{\tau(n)}\right)\left[\mu\left(B_{\tau(n)}\right)-\mu\left(B_{\tau(n+1)}\right)\right] \\
& =\sum_{i=1}^{n} v\left(\Delta \widetilde{A}_{i}\right)\left[\mu\left(B_{\tau(i)}\right)-\mu\left(B_{\tau(i+1)}\right)\right]
\end{aligned}
$$

where $v\left(\Delta \widetilde{A}_{\tau(1)}\right), v\left(\Delta \widetilde{A}_{\tau(2)}\right), \ldots, v\left(\Delta \widetilde{A}_{\tau(n)}\right)$ is the permutation of $v\left(\Delta \widetilde{A}_{1}\right), v\left(\Delta \widetilde{A}_{2}\right), \ldots, v\left(\Delta \widetilde{A}_{n}\right)$, for any $i, v\left(\Delta \widetilde{A}_{\tau(i)}\right) \leq$ $v\left(\Delta \widetilde{A}_{\tau(i+1)}\right) ; B_{\tau(i)}=\left(x_{\tau(i)}, x_{\tau(i+1)}, \ldots, x_{\tau(n)}\right)$ and $B_{\tau(n+1)}=0$.

\section{Decision-Making Method and Step}

MAGDM problem aims to select or sort alternatives with multiple attributes for a decision-making group. Suppose that $\mathrm{DM}=\left\{\mathrm{DM}_{1}, \mathrm{DM}_{2}, \ldots, \mathrm{DM}_{k}, \ldots, \mathrm{DM}_{h}\right\}, k=1,2, \ldots, h$ are decision makers who are invited to select the best alternative from $m$ alternatives $A_{i}(i=1,2, \ldots, m)$ assessed on $n$ attributes $C_{j}(j=1,2, \ldots, n)$.

Step 1. Obtain intuitionistic trapezoidal fuzzy decision matrixes given by decision makers, which can be denoted by

$$
\begin{gathered}
X^{1}=\left[\tilde{x}_{i j}^{1}\right]_{m \times n}, \\
X^{2}=\left[\tilde{x}_{i j}^{2}\right]_{m \times n}, \\
\vdots \\
X^{h}=\left[\tilde{x}_{i j}^{h}\right]_{m \times n},
\end{gathered}
$$

where $\tilde{x}_{i j}^{k}=\left(\left[a_{i j}^{k}, b_{i j}^{k}, c_{i j}^{k}, d_{i j}^{k}\right] ; \mu_{i j}^{k}, v_{i j}^{k}\right)$ is the performance of alternative $A_{i}$ under attribute $C_{j}$ provided by $\mathrm{DM}_{k}$ with ITFN.

Step 2. According to the prospect theory, decision makers give their different reference points $\widetilde{R}_{j}^{k}=\left(\left[a_{j}^{k}, b_{j}^{k}, c_{j}^{k}, d_{j}^{k}\right] ; \mu_{j}^{k}\right.$, $\left.v_{j}^{k}\right)$ based on different attributes $C_{j}(i=1,2, \ldots, n)$. According to Definition 9 , the deviation degree between $\widetilde{x}_{i j}^{k}$ and $\widetilde{R}_{j}^{k}$ is 


$$
\begin{aligned}
& \Delta \tilde{x}_{i j}^{k}=\tilde{x}_{i j}^{k} \ominus \widetilde{R}_{j}^{k} \\
& \quad=\left(\left[a_{i j}^{k}-d_{j}^{k}, b_{i j}^{k}-c_{j}^{k}, c_{i j}^{k}-b_{j}^{k}, d_{i j}^{k}-a_{j}^{k}\right] ; \mu_{\widehat{x}_{i j}^{k} \ominus R_{j}^{k}},\right. \\
& \left.\quad v_{\underline{x}_{i j} \ominus R_{j}^{k}}\right) .
\end{aligned}
$$

Step 3. According to Definition 11, intuitionistic trapezoidal fuzzy decision matrixes $X^{1}=\left[\tilde{x}_{i j}^{1}\right]_{m \times n}, X^{2}=\left[\tilde{x}_{i j}^{2}\right]_{m \times n}, \ldots$, $X^{h}=\left[\tilde{x}_{i j}^{h}\right]_{m \times n}$ are transformed to intuitionistic trapezoidal fuzzy decision prospect matrixes $\mathrm{PX}^{1}=\left[v\left(\Delta \tilde{x}_{i j}^{1}\right)\right]_{m \times n}, \mathrm{PX}^{2}=$ $\left[v\left(\Delta \tilde{x}_{i j}^{2}\right)\right]_{m \times n}, \ldots, \mathrm{PX}^{h}=\left[v\left(\Delta \widetilde{x}_{i j}^{h}\right)\right]_{m \times n}$ by the deviation degree, where

$$
\begin{aligned}
& v\left(\Delta \tilde{x}_{i j}^{k}\right)
\end{aligned}
$$

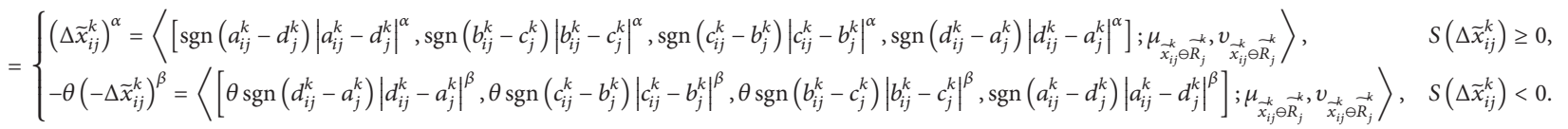

Step 4. By means of history sample information, the fuzzy measure is obtained. And then the intuitionistic trapezoidal fuzzy decision prospect matrixes are aggregated by the intuitionistic trapezoidal fuzzy Choquet integral prospect operator. The nuts and bolts are

$$
\begin{aligned}
& \int v\left(\Delta \tilde{x}_{i j}^{k}\right) d u \\
& =\operatorname{CITFCI}\left(v\left(\Delta \tilde{x}_{i j}^{1}\right), v\left(\Delta \tilde{x}_{i j}^{2}\right), \ldots, v\left(\Delta \tilde{x}_{i j}^{h}\right)\right) \\
& =v\left(\Delta \tilde{x}_{i j}^{\tau(1)}\right)\left[\mu\left(\mathrm{DM}_{\tau(1)}\right)-\mu\left(\mathrm{DM}_{\tau(2)}\right)\right] \oplus \cdots \\
& \quad \oplus v\left(\Delta \tilde{x}_{i j}^{\tau(h)}\right)\left[\mu\left(\mathrm{DM}_{\tau(h)}\right)-\mu\left(\mathrm{DM}_{\tau(h+1)}\right)\right] \\
& =\sum_{k=1}^{h} v\left(\Delta \tilde{x}_{i j}^{\tau(k)}\right)\left[\mu\left(\mathrm{DM}_{\tau(k)}\right)-\mu\left(\mathrm{DM}_{\tau(k+1)}\right)\right],
\end{aligned}
$$

where $v\left(\Delta \widetilde{x}_{i j}^{\tau(1)}\right), v\left(\Delta \widetilde{x}_{i j}^{\tau(2)}\right), \ldots, v\left(\Delta \widetilde{x}_{i j}^{\tau(h)}\right)$ is the permutation of $v\left(\Delta \tilde{x}_{i j}^{1}\right), v\left(\Delta \tilde{x}_{i j}^{2}\right), \ldots, v\left(\Delta \tilde{x}_{i j}^{h}\right)$. And then, the comprehensive prospect decision matrix CPX $=\left(\widetilde{\mathrm{cpx}}_{i j}\right)_{m \times n}$ is obtained.

$$
\begin{aligned}
& \operatorname{CPX}=\left(\widetilde{\mathrm{cpx}}_{i j}\right)_{m \times n} \\
& =\left[\operatorname{CITFNCI}\left(v\left(\Delta \tilde{x}_{i j}^{1}\right), v\left(\Delta \tilde{x}_{i j}^{2}\right), \ldots, v\left(\Delta \tilde{x}_{i j}^{h}\right)\right)\right]_{m \times n} \\
& = \begin{cases}\left(p a_{i j}, p b_{i j}, p c_{i j}, p d_{i j} ; p \mu_{i j}, p v_{i j}\right), & S\left(v\left(\Delta \tilde{x}_{i j}^{k}\right)\right) \geq 0, \\
\left(p a_{i j}^{\theta}, p b_{i j}^{\theta}, p c_{i j}^{\theta}, p d_{i j}^{\theta} ; p \mu_{i j}^{\theta}, p v_{i j}^{\theta}\right), & S\left(v\left(\Delta \tilde{x}_{i j}^{k}\right)\right)<0,\end{cases}
\end{aligned}
$$

where

$$
\begin{aligned}
& p a_{i j}=\sum_{k=1}^{h} \operatorname{sgn}\left(a_{i j}^{\tau(k)}-d_{j}^{\tau(k)}\right)\left|a_{i j}^{\tau(k)}-d_{j}^{\tau(k)}\right|^{\alpha} \Delta \mu_{\tau(k)}, \\
& p b_{i j}=\sum_{k=1}^{h} \operatorname{sgn}\left(b_{i j}^{\tau(k)}-c_{j}^{\tau(k)}\right)\left|b_{i j}^{\tau(k)}-c_{j}^{\tau(k)}\right|^{\alpha} \Delta \mu_{\tau(k)}, \\
& p c_{i j}=\sum_{k=1}^{h} \operatorname{sgn}\left(c_{i j}^{\tau(k)}-b_{j}^{\tau(k)}\right)\left|c_{i j}^{\tau(k)}-b_{j}^{\tau(k)}\right|^{\alpha} \Delta \mu_{\tau(k)}, \\
& p d_{i j}=\sum_{k=1}^{h} \operatorname{sgn}\left(d_{i j}^{\tau(k)}-a_{j}^{\tau(k)}\right)\left|d_{i j}^{\tau(k)}-a_{j}^{\tau(k)}\right|^{\alpha} \Delta \mu_{\tau(k)},
\end{aligned}
$$

$$
\begin{aligned}
& p \mu_{i j}=\frac{\sum_{k=1}^{h}\left[\left\|\Delta \tilde{x}_{i j}^{\tau(k)}\right\|\right]^{\alpha}\left(\mu_{x R}\right)_{\tau(k)}}{\sum_{k=1}^{h}\left[\left\|\Delta \tilde{x}_{i j}^{\tau(k)}\right\|\right]^{\alpha}}, \\
& p v_{i j}=\frac{\sum_{k=1}^{h}\left[\left\|\Delta \tilde{x}_{i j}^{\tau(k)}\right\|\right]^{\alpha}\left(v_{x R}\right)_{\tau(k)}}{\sum_{k=1}^{h}\left[\left\|\Delta \tilde{x}_{i j}^{\tau(k)}\right\|\right]^{\alpha}}, \\
& p a_{i j}^{\theta}=\sum_{k=1}^{h} \theta \operatorname{sgn}\left(d_{i j}^{\tau(k)}-a_{j}^{\tau(k)}\right)\left|d_{i j}^{\tau(k)}-a_{j}^{\tau \tau(k)}\right|^{\beta} \Delta \mu_{\tau(k)}, \\
& p b_{i j}^{\theta}=\sum_{k=1}^{h} \theta \operatorname{sgn}\left(c_{i j}^{\tau(k)}-b_{j}^{\tau(k)}\right)\left|c_{i j}^{\tau(k)}-b_{j}^{\tau(k)}\right|^{\beta} \Delta \mu_{\tau(k)}, \\
& p c_{i j}^{\theta}=\sum_{k=1}^{h} \theta \operatorname{sgn}\left(b_{i j}^{\tau(k)}-c_{j}^{\tau(k)}\right)\left|b_{i j}^{\tau(k)}-c_{j}^{\tau(k)}\right|^{\beta} \Delta \mu_{\tau(k)}, \\
& p d_{i j}^{\theta}=\sum_{k=1}^{h} \theta \operatorname{sgn}\left(a_{i j}^{\tau(k)}-d_{j}^{\tau(k)}\right)\left|a_{i j}^{\tau(k)}-d_{j}^{\tau(k)}\right|^{\beta} \Delta \mu_{\tau(k)}, \\
& p \mu_{i j}^{\theta}=\frac{\sum_{k=1}^{h}\left[-\theta\left\|\Delta \tilde{x}_{i j}^{\tau(k)}\right\|\right]^{\beta}\left(\mu_{x R}\right)_{\tau(k)}}{\sum_{k=1}^{h}\left[-\theta\left\|\Delta \tilde{x}_{i j}^{\tau(k)}\right\|\right]^{\beta}}, \\
& \Delta \mu_{\tau(k)}=\frac{\sum_{k=1}^{h}\left[-\theta\left\|\Delta \tilde{x}_{i j}^{\tau(k)}\right\|\right]^{\beta}\left(\mu_{x R}\right)_{\tau(k)}}{\sum_{k=1}^{h}\left[-\theta\left\|\Delta \tilde{x}_{i j}^{\tau(k)}\right\|\right]^{\beta}}, \\
& \left.v_{\tau(k)}\right)-\mu\left(H_{\tau(k+1)}\right), i=1,2, \ldots, m, j=1,2, \ldots, n .
\end{aligned}
$$

Step 5. Normalize the comprehensive prospect decision matrix.

$$
\begin{aligned}
& \operatorname{NCPX}=\left(\widetilde{\operatorname{ncpx}}{ }_{i j}\right)_{m \times n} \\
& =\left[\operatorname{NCITFNCI}\left(v\left(\Delta \tilde{x}_{i j}^{1}\right), v\left(\Delta \widetilde{x}_{i j}^{2}\right), \ldots, v\left(\Delta \widetilde{x}_{i j}^{h}\right)\right)\right]_{m \times n} \\
& = \begin{cases}\left(\frac{p a_{i j}}{D_{j}}, \frac{p b_{i j}}{D_{j}}, \frac{p c_{i j}}{D_{j}}, \frac{p d_{i j}}{D_{j}} ; p \mu_{i j}, p v_{i j}\right), \quad S\left(v\left(\Delta \widetilde{x}_{i j}^{k}\right)\right) \geq 0, \\
\left(\frac{p a_{i j}^{\theta}}{D_{j}^{\theta}}, \frac{p b_{i j}^{\theta}}{D_{j}^{\theta}}, \frac{p c_{i j}^{\theta}}{D_{j}^{\theta}}, \frac{p d_{i j}^{\theta}}{D_{j}^{\theta}} ; p \mu_{i j}^{\theta}, p v_{i j}^{\theta}\right), \quad S\left(v\left(\Delta \widetilde{x}_{i j}^{k}\right)\right)<0\end{cases} \\
& =\left(p a_{i j}^{*}, p b_{i j}^{*}, p c_{i j}^{*}, p d_{i j}^{*} ; p \mu_{i j}^{*}, p v_{i j}^{*}\right),
\end{aligned}
$$


where $D_{j}=\left(\sum_{i=1}^{m}\left[\left(p a_{i j}\right)^{2}+\left(p b_{i j}\right)^{2}+\left(p c_{i j}\right)^{2}+\left(p d_{i j}\right)^{2}\right]\right)^{1 / 2}$ and

$$
\begin{aligned}
& D_{j}^{\theta} \\
& =\left(\sum_{i=1}^{m}\left[\left(p a_{i j}^{\theta}\right)^{2}+\left(p b_{i j}^{\theta}\right)^{2}+\left(p c_{i j}^{\theta}\right)^{2}+\left(p d_{i j}^{\theta}\right)^{2}\right]\right)^{1 / 2}, \\
& \quad i=1,2, \ldots, m, j=1,2, \ldots, n .
\end{aligned}
$$

Step 6. Calculate the distance between the normalized comprehensive prospect decision information $\widetilde{\mathrm{ncpx}}_{i j}$ and the normalized ideal reference vector $R^{*}=([1,1,1,1] ; 1,0)$.

$$
\begin{aligned}
d_{i j} & =\frac{1}{8}\left(\left|\left(1+p \mu_{i j}^{*}-p v_{i j}^{*}\right) p a_{i j}^{*}-2\right|\right. \\
& +\left|\left(1+p \mu_{i j}^{*}-p v_{i j}^{*}\right) p b_{i j}^{*}-2\right| \\
& +\left|\left(1+p \mu_{i j}^{*}-p v_{i j}^{*}\right) p c_{i j}^{*}-2\right| \\
& \left.+\left|\left(1+p \mu_{i j}^{*}-p v_{i j}^{*}\right) p d_{i j}^{*}-2\right|\right) .
\end{aligned}
$$

Step 7. Calculate the grey correlation coefficient $r_{i j}$ :

$$
r_{i j}=\frac{d_{\min }+0.5 * d_{\max }}{d_{i j}+0.5 * d_{\max }},
$$

where $d_{\max }=\max \left\{d_{i j}\right\}, d_{\min }=\min \left\{d_{i j}\right\}$.

Step 8. Construct the optimization model to obtain the best projection vector $\omega_{j}$.

$$
\begin{array}{ll}
\max & \sum_{j=1}^{n} \omega_{j} r_{i j}, \\
\text { s.t. } & \sum_{j=1}^{n} \omega_{j}^{2}=1, \quad \omega_{j}^{2} \geq 0 .
\end{array}
$$

Step 9. Calculate the grey correlation projection value $z_{i}$.

Draw the experience from [14]; the cosine vector of the projection angle between the alternative $A_{i}$ and the normalized ideal reference vector is

$$
\chi_{i}=\cos \alpha=\frac{\sum_{j=1}^{n} \omega_{j} r_{i j} \omega_{j}}{\sqrt{\sum_{j=1}^{n}\left[\omega_{j} r_{i j}\right]^{2}} \sqrt{\sum_{j=1}^{n} \omega_{j}^{2}}} .
$$

Obviously, $0 \leq d_{i} \leq 1$, the bigger the better.

The grey correlation projection value $z_{i}$ between the alternative $A_{i}$ and the normalized ideal reference vector is

$$
z_{i}=M\left(A_{i}\right) \cdot \chi_{i}=\frac{\sum_{j=1}^{n} \omega_{j}^{2} r_{i j}}{\sqrt{\sum_{j=1}^{n} \omega_{j}^{2}}}=\sum_{j=1}^{n} \bar{\omega}_{j} r_{i j},
$$

where $M\left(A_{i}\right)=\sum_{j=1}^{n}\left[\omega_{j} r_{i j}\right]^{2}$ is the moduli of $A_{i}$. And $\bar{\omega}_{j}$ is the grey correlation projection vector, and it can be calculated by

$$
\bar{\omega}_{j}=\frac{\omega_{j}^{2}}{\sqrt{\sum_{j=1}^{n} \omega_{j}^{2}}} .
$$

According to the grey correlation projection value $z_{i}$, we can rank the alternatives.

\section{Case Analysis}

5.1. An Example and Analysis Process. Jinzhong power supply company in ShanXi province plans to select one of the four equipment suppliers $A=\left\{A_{1}, A_{2}, A_{3}, A_{4}\right\}$ for an engineering project cooperation. There are four attributes in this case, including the product quality $C_{1}$, technology capability $C_{2}$, pollution control $C_{3}$, and environment management $C_{4}$, which are all benefit oriented attributes. The company invites three experts to form a group of decision makers. $\mathrm{DM}_{1}$ is from the production department; $\mathrm{DM}_{2}$ is from the operation and maintenance department; $\mathrm{DM}_{3}$ is from the quality inspection department.

Step 1. Three experts $\left\{\mathrm{DM}_{1}, \mathrm{DM}_{2}, \mathrm{DM}_{3}\right\}$ are invited to select the best alternative. Tables 1-3 show the experts' evaluation information with intuitionistic trapezoidal fuzzy numbers with respect to attributes under alternatives.

Step 2. Three experts provide reference points according to the four attributes, which are shown in Table 4.

Step 3. Set $\alpha=0.89, \beta=0.92, \sigma=2.25$ [15] and calculate the prospect value function by (19). Then we transform the intuitionistic trapezoidal fuzzy decision matrixes to intuitionistic trapezoidal fuzzy prospect matrixes. Due to space limitation, the calculation process is omitted.

Step 4. The fuzzy measures are obtained according to history sample information. $\mu(\varnothing)=0, \mu\left(\left\{\mathrm{DM}_{1}\right\}\right)=0.4$, $\mu\left(\left\{\mathrm{DM}_{2}\right\}\right)=0.5, \mu\left(\left\{\mathrm{DM}_{1}\right\}\right)=0.6, \mu\left(\left\{\mathrm{DM}_{1}, \mathrm{DM}_{2}\right\}\right)=0.7$, $\mu\left(\left\{\mathrm{DM}_{1}, \mathrm{DM}_{3}\right\}\right)=0.7, \mu\left(\left\{\mathrm{DM}_{3}, \mathrm{DM}_{2}\right\}\right)=0.8$, and $\mu\left(\left\{\mathrm{DM}_{1}\right.\right.$, $\left.\left.\mathrm{DM}_{2}, \mathrm{DM}_{3}\right\}\right)=1$. According to (20), the comprehensive prospect decision matrix is obtained shown in Table 5.

Step 5. Normalize the comprehensive prospect decision matrix based on (23). The result is shown in Table 6.

Steps 6-7. By (25)-(26), the distance and grey correlation coefficients are calculated shown in Table 7.

Step 8. Set the parameters of the optimization model, $m=4$ and $n=4$, and the cluster is divided into two categories. Then the best projection vector is $\omega_{j}=(0.5745,0.2647,0.6622$, 0.4017).

Step 9. By (30), the grey correlation projection vector $\bar{\omega}_{j}=$ $(0.3305,0.070,0.4385,0.1613)$ is obtained. The grey correlation projection values of alternatives are computed: $z_{1}=$ $0.8698, z_{2}=0.8684, z_{3}=0.9342$, and $z_{4}=0.9189$. The ranking result is $A_{3}>A_{4}>A_{1}>A_{2}$, so the best alternative is $A_{3}$.

5.2. Comparison Analysis with Other Methods. The goal of decision-making in the real world is typically to find the optimal alternative from a group of alternatives. Lots of methods have been applied to MADM to make the result 
TABLE 1: The evaluation information from $\mathrm{DM}_{1}$.

\begin{tabular}{lcccc}
\hline & $C_{1}$ & $C_{2}$ & $C_{3}$ & $C_{4}$ \\
\hline$A_{1}$ & $([3,4,5,8] ; 0.7,0.2)$ & $([3,4,6,8] ; 0.6,0.2)$ & $([1,3,6,7] ; 0.8,0.1)$ & $([2,3,5,7] ; 0.7,0.2)$ \\
$A_{2}$ & $([1,2,3,5] ; 0.5,0.3)$ & $([2,4,7,9] ; 0.6,0.3)$ & $([1,3,4,5] ; 0.6,0.3)$ & $([4,5,7,8] ; 0.8,0.1)$ \\
$A_{3}$ & $([2,3,4,6] ; 0.8,0.1)$ & $([4,6,8,9] ; 0.7,0.1)$ & $([2,5,6,8] ; 0.5,0.3)$ & $([6,7,8,9] ; 0.8,0.1)$ \\
$A_{4}$ & $([3,5,7,9] ; 0.6,0.2)$ & $([3,5,6,7] ; 0.8,0.1)$ & $([2,3,4,6] ; 0.7,0.2)$ & $([1,3,5,7] ; 0.7,0.1)$ \\
\hline
\end{tabular}

TABLE 2: The evaluation information from $\mathrm{DM}_{2}$.

\begin{tabular}{lcccc}
\hline & $C_{1}$ & $C_{2}$ & $C_{3}$ & $C_{4}$ \\
\hline$A_{1}$ & $([1,4,6,9] ; 0.6,0.3)$ & $([2,3,4,7] ; 0.7,0.1)$ & $([3,4,6,8] ; 0.7,0.2)$ & $([1,3,5,8] ; 0.6,0.2)$ \\
$A_{2}$ & $([3,4,7,8] ; 0.6,0.2)$ & $([3,4,5,6] ; 0.5,0.3)$ & $([4,6,8,9] ; 0.7,0.1)$ & $([1,5,7,9] ; 0.7,0.2)$ \\
$A_{3}$ & $([2,6,7,8] ; 0.7,0.1)$ & $([1,3,4,8] ; 0.6,0.2)$ & $([2,3,5,6] ; 0.5,0.4)$ & $([3,4,6,8] ; 0.7,0.2)$ \\
$A_{4}$ & $([2,5,6,7] ; 0.7,0.2)$ & $([2,5,7,9] ; 0.8,0.1)$ & $([3,5,6,7] ; 0.7,0.2)$ & $([2,3,5,8] ; 0.6,0.1)$ \\
\hline
\end{tabular}

TABLE 3: The evaluation information from $\mathrm{DM}_{3}$.

\begin{tabular}{lcccc}
\hline & $C_{1}$ & $C_{2}$ & $C_{3}$ & $C_{4}$ \\
\hline$A_{1}$ & $([3,5,7,8] ; 0.8,0.1)$ & $([1,4,5,9] ; 0.5,0.3)$ & $([2,4,6,8] ; 0.7,0.1)$ & $([4,6,7,8] ; 0.7,0.2)$ \\
$A_{2}$ & $([1,6,7,9] ; 0.7,0.2)$ & $([3,4,6,8] ; 0.6,0.1)$ & $([2,5,6,9] ; 0.8,0.1)$ & $([1,2,3,4] ; 0.4,0.2)$ \\
$A_{3}$ & $([2,4,7,8] ; 0.6,0.2)$ & $([3,6,7,8] ; 0.6,0.2)$ & $([1,2,5,7] ; 0.7,0.2)$ & $([4,6,7,10] ; 0.7,0.1)$ \\
$A_{4}$ & $([3,5,6,7] ; 0.7,0.2)$ & $([2,3,5,8] ; 0.5,0.4)$ & $([4,6,7,8] ; 0.8,0.1)$ & $([5,7,8,9] ; 0.6,0.2)$ \\
\hline
\end{tabular}

more accurate or less biased. Some scholars consider several appropriate ways to select the optimal alternative based on the majority principle. But this suggestion assumes that all methods are appropriate for the decision-making problem. Table 8 shows the comparison with other methods.

$\mathrm{Wu}$ and Cao use the ITFN weighted geometric operator and hybrid geometric operator to obtain the collective overall values and calculate the distances between collective overall values and positive solution. Finally, the ranking result is $A_{3}>A_{1}>A_{4}>A_{2}$. Wu and Cao's method is only suitable for the case where the weights of attributes and decision makers are known. Therefore, they cannot solve the MAGDM problems with unknown weights, whereas the proposed method aggregates decision makers' data on bias of attribute independence and decision makers' risk preference. This implies that the proposed method has wider real application range than the method in [8]. It shows from Tables $1-3$ that there are too large $([6,7,8,9] ; 0.8,0.1)$ for $A_{3}$ on $C_{4}$ in Table 1 and too small $([1,2,3,4] ; 0.4,0.2)$ for $A_{2}$ on $C_{4}$ in Table 3, which results in the ranking order $A_{3}>$ $A_{1}>A_{4}>A_{2}$ by Wu and Cao's method. The two operators developed by [8] cannot reflect the influence of unfair data (too large or too small ITFNs), whereas, the proposed method can effectively relieve the influence of unfair data by assigning low weights to those unfair data.

Wan's method includes four cases, and we choose the first case that the weight vectors of attributes are unknown. The collective results are $m_{\mu}\left(A_{1}\right)=0.2241, m_{\mu}\left(A_{2}\right)=$ $0.1123, m_{\mu}\left(A_{3}\right)=0.2539$, and $m_{\mu}\left(A_{4}\right)=0.1786$, which are calculated by TrIFPG operator and linear goal programming model. Although the ranking result is the same as this paper's result, the aggregation operator in Wan's paper is based on power geometric operator and has an obvious drawback. For example, there are three ITFNs: $([0,2,3,4] ; 0.15,0.8)$, $([6,7,9,10] ; 0.8,0)$, and $([6,7,8,9] ; 0.9,0.05)$. After being aggregated by TrIFPG or TrIFPHG operator, there will be $a=$ 0 due to the fact that there only exists $([0,2,3,4] ; 0.15,0.8)$ no matter what the next ITFNs are. All multiplication aggregation operators without considering the other values will get the wrong results when we meet the maximum or minimum data.

Xu's method aims at IFNs, so we could only use the membership and nonmembership degree in Tables 1-3 to calculate the result. Xu utilized the intuitionistic fuzzy power weighted geometric operator and intuitionistic fuzzy weighted geometric operator to deal with IFNs. After computation, the overall preference values of alternatives are obtained as

$$
\begin{aligned}
& r_{1}=(0.4967,0.3217), \\
& r_{2}=(0.5015,0.2324), \\
& r_{3}=(0.6349,0.2317), \\
& r_{4}=(0.5267,0.2106) .
\end{aligned}
$$

The scores of $r_{i}(i=1,2,3,4)$ are obtained as $s_{1}=0.175, s_{2}=$ $0.2691, s_{3}=0.4023$, and $s_{4}=0.3161$. The ranking order is $A_{3}>A_{4}>A_{2}>A_{1}$, which is similar to this paper's ranking result. But Xu's method studies the ones for IFNs. Since ITFNs are the combination of trapezoidal fuzzy numbers with IFNs, ITFNs can greatly enhance the representation ability of information than IFNs. 


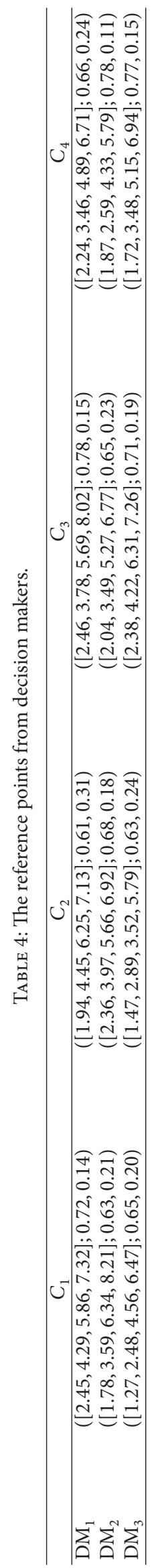




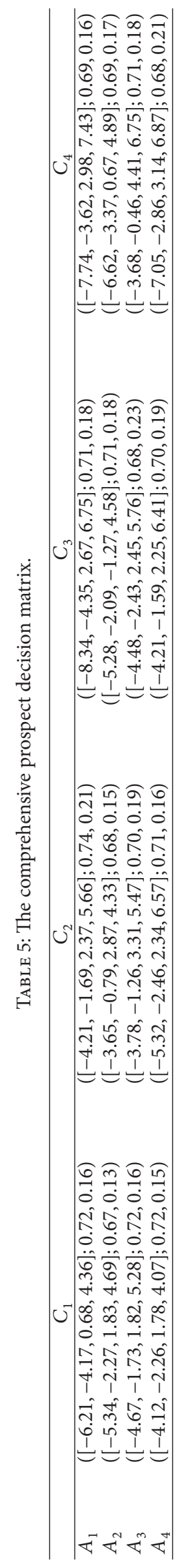




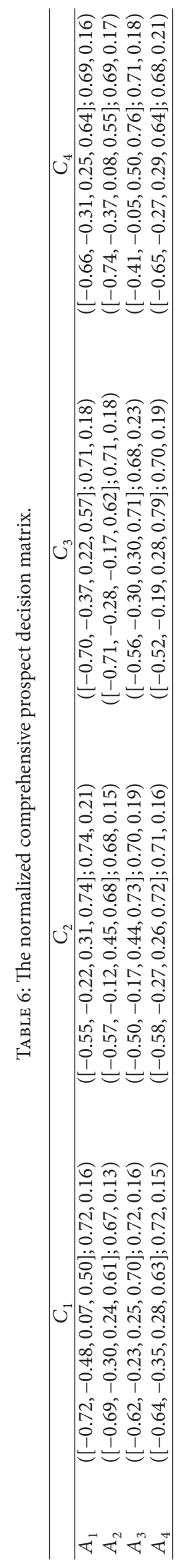


TABLE 7: The distance and grey correlation coefficients.

\begin{tabular}{ccccccccc}
\hline & & \multicolumn{2}{c}{$d_{i j}$} & & & $r_{i j}$ & \\
& $C_{1}$ & $C_{2}$ & $C_{3}$ & $C_{4}$ & $C_{1}$ & $C_{2}$ & $C_{3}$ \\
\hline$A_{1}$ & 1.120 & 0.947 & 1.053 & 1.016 & 0.839 & 0.935 & 0.874 \\
$A_{2}$ & 1.027 & 0.917 & 1.105 & 1.094 & 0.887 & 0.953 & 0.846 \\
$A_{3}$ & 0.981 & 0.906 & 0.971 & 0.849 & 0.914 & 0.961 & 0.920 \\
$A_{4}$ & 1.016 & 0.976 & 0.934 & 0.998 & 0.894 & 0.917 & 0.952 \\
\hline
\end{tabular}

TABLE 8: A comparison of the preference orders of the alternatives of different methods.

\begin{tabular}{ll}
\hline Methods & Preference order \\
\hline Wu and Cao [8] & $A_{3}>A_{1}>A_{4}>A_{2}$ \\
Wan and Dong [9] & $A_{3}>A_{1}>A_{4}>A_{2}$ \\
Xu [12] & $A_{3}>A_{4}>A_{2}>A_{1}$ \\
This paper & $A_{3}>A_{4}>A_{1}>A_{2}$ \\
\hline
\end{tabular}

\section{Conclusion}

Intuitionistic trapezoidal fuzzy number is suitable for expressing the fuzziness and uncertainty of the decision information in complex MAGDM problems. In the light of the faults in ITFN's operation rules, this paper redefines some basic operation rules of ITFN. In order to eliminate the interdependency in attributes, the intuitionistic trapezoidal fuzzy Choquet integral is defined. In consideration of decision makers' bounded rationality, the intuitionistic trapezoidal fuzzy Choquet integral prospect (ITFCIP) operator is defined to aggregate the ITFNs. Aiming at MAGDM problem with trapezoidal intuitionistic fuzzy numbers under unknown attribute weights, a grey relation-projection pursuit dynamic cluster method based on ITFCIP operator is presented. This method defines the prospect value function based on ITFNs, obtains the fuzzy measure according to the history sample information, and then constructs the comprehensive prospect decision matrix based on ITFCIP operator. In addition, a grey relation-projection pursuit dynamic cluster method is proposed to select the best alternative. This combination improves grey correlation projection method under the comprehensive consideration of preferential membership and grey correlation. The firefly algorithm is used to optimize the objective function of projection for obtaining the best projection direction of grey correlation projection values, and the grey correlation projection values are evaluated, which are applied to classify, rank, and prefer the alternatives.

\section{Competing Interests}

The authors declare that there is no conflict of interests regarding the publication of this manuscript.

\section{Acknowledgments}

The authors would like to acknowledge the supports by National Natural Science Foundation of China (nos. 71271084, 71671065), the Fundamental Research Funds for
Central Universities (2016XS74), and the technology project of state grid (KJGW2015-020).

\section{References}

[1] A. Hatami-Marbini and M. Tavana, "An extension of the Electre I method for group decision-making under a fuzzy environment," Omega, vol. 39, no. 4, pp. 373-386, 2011.

[2] F. Shen, J. Xu, and Z. Xu, "An outranking sorting method for multi-criteria group decision making using intuitionistic fuzzy sets," Information Sciences, vol. 334-335, pp. 338-353, 2016.

[3] B. Liu, Y. Shen, W. Zhang, X. Chen, and X. Wang, "An interval-valued intuitionistic fuzzy principal component analysis model-based method for complex multi-attribute largegroup decision-making," European Journal of Operational Research, vol. 245, no. 1, pp. 209-225, 2015.

[4] S.-M. Chen, S.-H. Cheng, and W.-H. Tsai, "Multiple attribute group decision making based on interval-valued intuitionistic fuzzy aggregation operators and transformation techniques of interval-valued intuitionistic fuzzy values," Information Sciences, vol. 367-368, pp. 418-442, 2016.

[5] J. Yuan and C. Li, "A new method for multi-attribute decision making with intuitionistic trapezoidal fuzzy random variable," International Journal of Fuzzy Systems, pp. 1-12, 2016.

[6] G. N. V. Lakshmana, S. Jeevaraj, and P. Dhanasekaran, "A linear ordering on the class of Trapezoidal intuitionistic fuzzy numbers," Expert Systems with Applications, vol. 60, pp. 269279, 2016.

[7] G. Kumar, R. K. Bajaj, and N. Gandotra, "Algorithm for shortest path problem in a network with interval-valued intuitionistic trapezoidal fuzzy number," Procedia Computer Science, vol. 70, pp. 123-129, 2015.

[8] J. Wu and Q.-W. Cao, "Same families of geometric aggregation operators with intuitionistic trapezoidal fuzzy numbers," Applied Mathematical Modelling, vol. 37, no. 1-2, pp. 318-327, 2013.

[9] S.-P. Wan and J.-Y. Dong, "Power geometric operators of trapezoidal intuitionistic fuzzy numbers and application to multiattribute group decision making," Applied Soft Computing, vol. 29, pp. 153-168, 2015.

[10] J. Yuan, C. Li, F. Xu, B. Sun, and W. Li, "A group decision making approach in interval-valued intuitionistic hesitant fuzzy environment with confidence levels," Journal of Intelligent \& Fuzzy Systems, vol. 31, no. 3, pp. 1909-1919, 2016.

[11] S.-M. Chen and C.-H. Chang, "Fuzzy multiattribute decision making based on transformation techniques of intuitionistic fuzzy values and intuitionistic fuzzy geometric averaging operators," Information Sciences, vol. 352-353, pp. 133-149, 2016.

[12] Z. Xu, "Approaches to multiple attribute group decision making based on intuitionistic fuzzy power aggregation operators," Knowledge-Based Systems, vol. 24, no. 6, pp. 749-760, 2011. 
[13] Z.-S. Chen and Y.-L. Li, "Approach for group MULTIMOORA decision making based upon prospect intuitionistic trapezoidal fuzzy number Choquet integral operator," Control and Decision, vol. 29, no. 6, pp. 1053-1063, 2014.

[14] Y.-C. Gong, Z.-Y. Ren, F. Ding et al., “Grey relation-projection pursuit dynamic cluster method for multiattribute decision making assessment with trapezoidal intuitionistic fuzzy numbers," Control and Decision, vol. 30, no. 7, pp. 1333-1339, 2015.

[15] Z.-S. Chen and Y.-L. Li, "An improved MULTIMOORA approach for group decision making based upon interdependent inputs of intuitionistic trapezoidal fuzzy numbers," System Engineering Theory \& Practice, vol. 34, no. 12, pp. 3138-3156, 2014. 


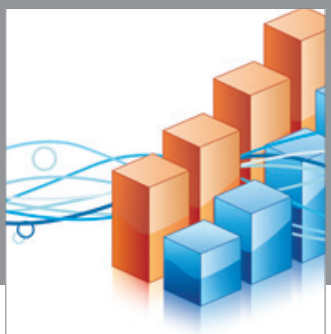

Advances in

Operations Research

vatem alat4

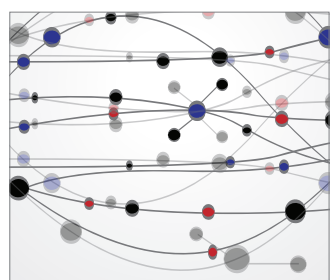

\section{The Scientific} World Journal
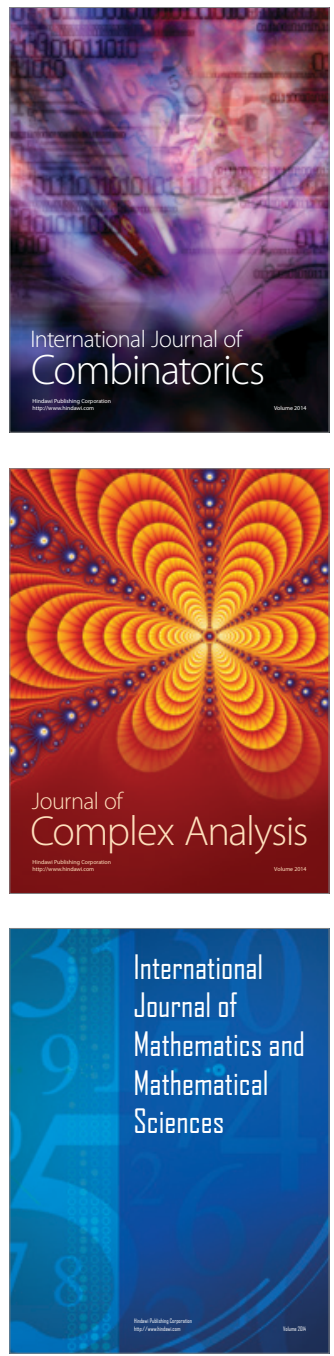
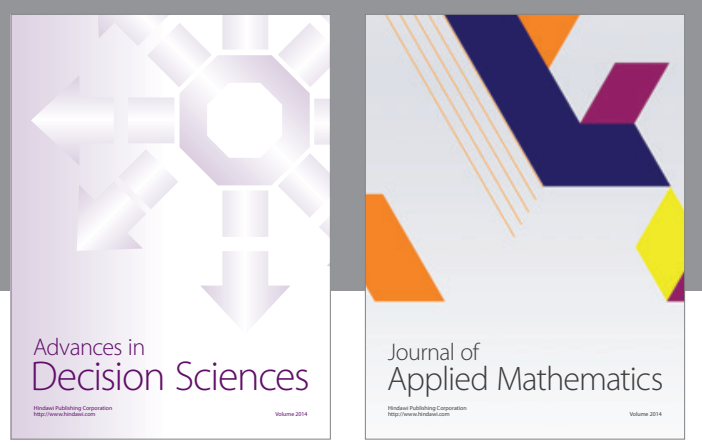

Algebra

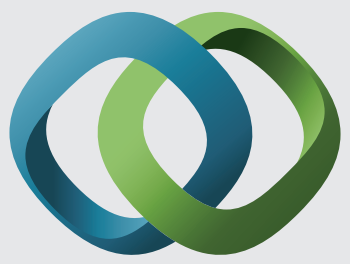

\section{Hindawi}

Submit your manuscripts at

https://www.hindawi.com
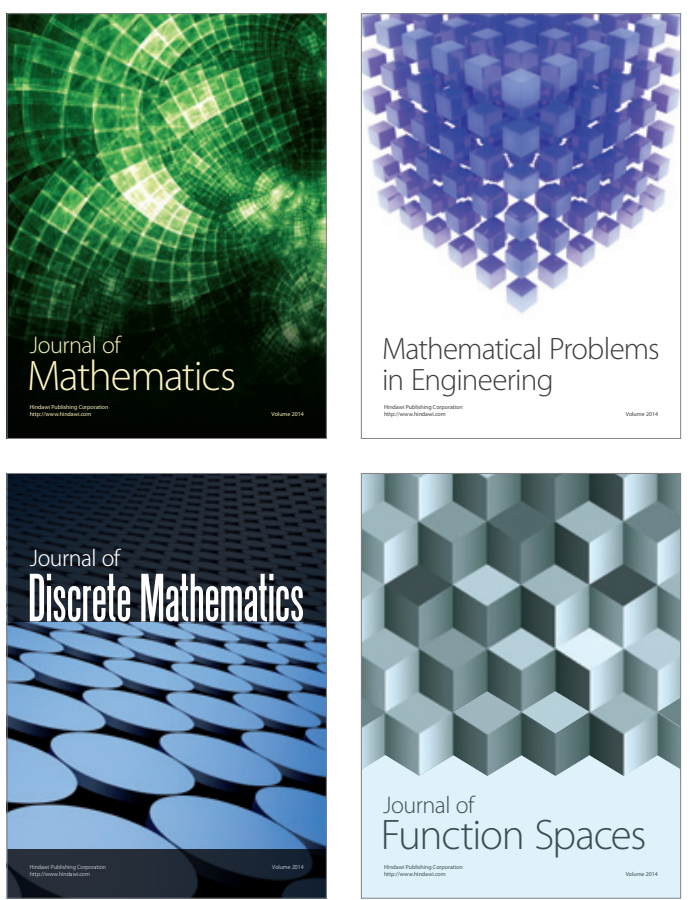

Mathematical Problems in Engineering
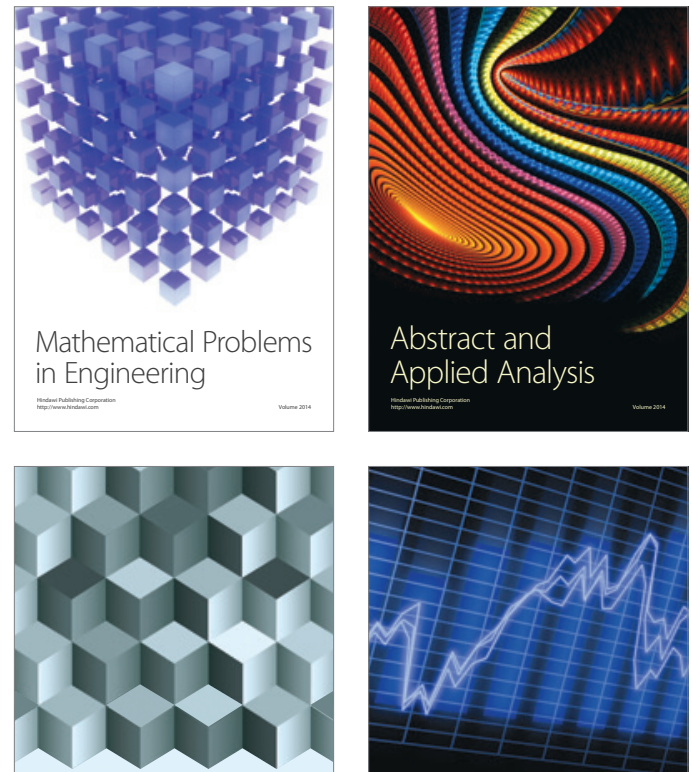

Journal of

Function Spaces

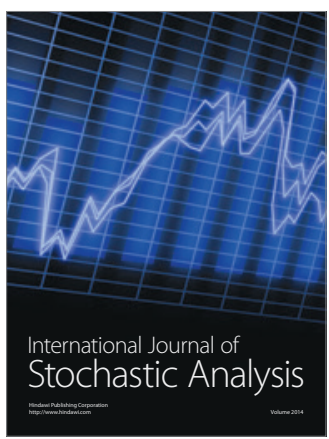

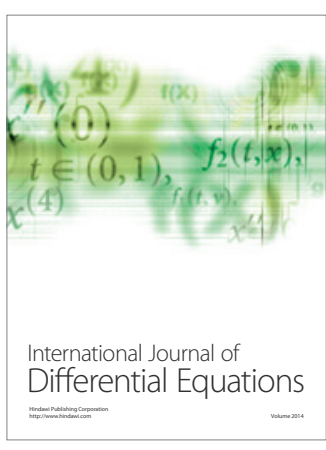
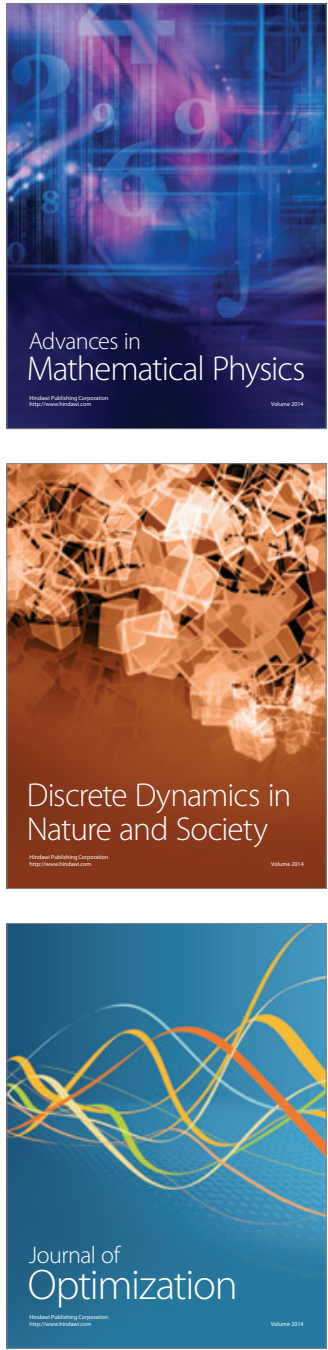